\title{
Conjugation of a self-antigen to papillomavirus-like particles allows for efficient induction of protective autoantibodies
}

\author{
Bryce Chackerian, Douglas R. Lowy, and John T. Schiller \\ Laboratory of Cellular Oncology, NIH, Bethesda, Maryland, USA \\ Address correspondence to: John T. Schiller, Laboratory of Cellular Oncology, National Institute of Health, \\ Building 36, Room 1D-32, Bethesda, Maryland 20892-4040, USA. \\ Phone: (301) 496-6539; Fax: (301) 480-5322; E-mail: schillej@dc37a.nci.nih.gov.
}

Received for publication November 27, 2000, and accepted in revised form June 25, 2001.

\begin{abstract}
High avidity and long-lasting autoantibodies to a self-polypeptide (TNF- $\alpha$ ) were generated after parenteral vaccination of mice with low doses of virus-like particle-based (VLP-based) vaccines that were constructed by linking mouse TNF- $\alpha$ peptides to the surface of papillomavirus VLPs. High-titer autoantibodies were induced with or without coadministration of potent conventional adjuvants, but were enhanced by coadministration of CFA. Compared with immunization with the fusion protein alone, attachment to VLPs increased autoantibody titers 1,000-fold. A comparison of Ab responses against the self (TNF- $\alpha$ ) and foreign components of the fusion protein showed that VLP conjugation abrogated the ability of the humoral immune system to distinguish between self and foreign. Similar levels of IgM were detected to self and foreign epitopes regardless of the assembly state of the antigen, suggesting that conjugation of self-peptides to VLPs promotes survival or expansion of mature autoreactive B cells. In a mouse model, vaccination with conjugated particles inhibited development of type II collagen-induced arthritis. Together, these results suggest a potentially flexible method to efficiently generate autoantibodies against specific self-proteins that mediate arthritis and other diseases.
\end{abstract}

J. Clin. Invest. 108:415-423 (2001). DOI:10.1172/JCI200111849.

\section{Introduction}

Practical vaccine strategies to induce potent $\mathrm{Ab}$ responses to self-antigens remain to be developed. Such strategies could prove efficacious in the growing number of diseases for which mAb-based therapies have produced encouraging results in animal studies and clinical trials. Ab-based strategies hold the potential to treat a variety of human diseases: for example, Abs targeting ErbB-2 (1), $\beta$-amyloid (2), and $\operatorname{IgE}(3)$ have been proposed to treat breast cancer, Alzheimer disease, and allergy, respectively. Vaccines that induce autoantibodies to disease targets would have several theoretical advantages over $m A b$ therapy. The levels of therapeutic Ab's would likely be more constant over time, potentially increasing effectiveness, treatment could be administered much less frequently, and there would be less likelihood for the development of an inactivating $\mathrm{Ab}$ response to the therapeutic Ab's.

The mammalian immune system normally fails to generate Ab's against self-proteins. However, B cell tolerance is not rigorous, and potentially self-reactive $\mathrm{B}$ cells are continuously generated as a result of recombinase-activating gene reactivation and peripheral editing of B cell receptor genes (4-6). Indeed, naturally occurring autoantibodies may arise by molecular mimicry by microbial agents, and this process has been implicated, with varying degrees of proof, in the pathogenesis of several mammalian autoimmune diseases (for review, see ref. 7). Experimentally, the chal- lenge is to develop vaccines suitable for human use that promote class switching, multiplication, affinity maturation, and survival of autoreactive $B$ cells. Autoantibodies have been induced by DNA immunization, but only after the appearance of autoimmune disease $(8,9)$. In healthy animals two broad experimental strategies have been used: immunization with self-antigen chemically conjugated or genetically linked to foreign Th epitopes in the presence of a strong adjuvant (10-15) and immunization with selfantigen arrayed in an ordered fashion on the surface of a virus-like particle (VLP) (16). The relative contributions of linked $\mathrm{T}$ cell help, ordered structure, and conventional adjuvants in the induction of mature autoreactive B cells have not been well characterized.

Based on data indicating that highly repetitive antigen structures strongly initiate B cell responses (17), we hypothesized previously that autoantibodies could be generated by presenting a target self-peptide within a highly organized context as part of the regular array of assembled capsomeres on a virus particle. Papillomavirus VLPs are nonenveloped icosahedral structures that consist of a regular array of 72 pentameric capsomeres composed of the major capsid protein, L1 $(18,19)$. Papillomavirus VLPs have the ability to induce strong B cell responses at low doses without adjuvant and are the basis for papillomavirus vaccines now being tested in clinical trials $(20,21)$. In a previous study, we genetically inserted an extracellular 
loop of mouse CCR5 into an immunodominant region of the $\mathrm{L} 1$ gene of bovine papillomavirus (BPV) to generate chimeric L1-CCR5 VLPs. Inoculation of chimeric L1-CCR5 particles into mice induced hightiter anti-CCR5 autoantibodies that bound to the receptor and inhibited chemokine binding and HIV infection in vitro (16). However, subsequent efforts to generate additional chimeric L1 VLPs incorporating different self-peptides proved problematic. We found that the ability of different chimeric L1-self-peptide chimeras to self-assemble into VLPs was highly unpredictable, limiting the general applicability of this technique (our unpublished observations). To circumvent this problem, we have now developed a more flexible and reliable approach in which self-antigens are conjugated to preformed VLPs. This technique takes advantage of the strong interaction between biotinylated VLPs and a streptavidin-self-peptide (SA-selfpeptide) fusion protein.

As a model target self-antigen we chose TNF- $\alpha$, a proinflammatory cytokine that plays a key role in the pathogenesis of a number of chronic inflammatory diseases $(22,23)$. In human clinical trials, modulation of TNF- $\alpha$ activity using a mAb effectively reduced the symptoms of rheumatoid arthritis and Crohn disease (24). Here, we demonstrate that immunization of mice with VLPs conjugated to a SA-mouse TNF- $\alpha$ fusion protein induces high-titer and long-lasting TNF- $\alpha$ autoantibodies that block the development of collageninduced arthritis. Furthermore, using this system we demonstrate that presentation of self-antigen on papillomavirus VLPs, but not simple linkage to foreign peptides in the presence of a potent conventional adjuvant, can abrogate the distinction between foreign and self in the induction of systemic IgG Abs.

\section{Methods}

Generation of SA-TNF- $\alpha$ clones. Two nucleotide sequences predicted to encode amino acids (aa) 3-22 (SSQNSSDKPVAHVVANHQVE) and 29-47 (SQRANALLANGMDLKDNQL) of the mature form of mouse TNF- $\alpha$ were made using an Applied Biosystems DNA synthesizer (Foster City, California, USA). Nucleotide sequence predicted to encode aa 3-54 (SSQNSSDKPVAHVVANHQVEEQLEWLSQRANALLANGMDLKDNQLVVPADGL) of mouse TNF- $\alpha$ was amplified by PCR from a cDNA library generated from LPS-stimulated J774 cells (as described by ref. 25). Each sequence was engineered to contain an EcoRI restriction site at its $5^{\prime}$ end and a stop codon followed by a BamHI restriction site at its $3^{\prime}$ end. The sequences were cloned using the restriction sites as a fusion partner with SA in the expression vector PTSA-18F (26). Clones were verified by sequence analysis.

Expression and purification of $S A-T N F-\alpha$ clones. SA-TNF- $\alpha$ constructs were transformed into lysogen BL21(DE2)(pLysS) (Novagen, Madison, Wisconsin, USA) for expression. The inclusion body fraction was prepared from IPTG-induced cultures as described (27), dissolved in $6 \mathrm{M}$ guanidine hydrochloride ( $\mathrm{pH} 1.5$ ), and then dialyzed (two times) against $0.2 \mathrm{M}$ sodium bicarbonate ( $\mathrm{pH}$ 8.7). Clarified dialysate was concentrated using a Filtron $10 \mathrm{~K}$ centrifugal concentrator (Pall Filtron Corp., Northborough, Massachusetts, USA). In some cases $S A$ was purified to homogeneity by applying the dialysate to a 2-iminobiotin-agarose column (Pierce Chemical Co., Rockford, Illinois, USA), as described (26).

Biotin ELISA. The active concentration of SA-TNF- $\alpha$ fusion proteins was determined by ELISA by measuring reactivity to biotinylated BSA (Pierce Chemical Co.) or biotinylated VLPs immobilized (at $200 \mathrm{ng} /$ well) on Immulon II microtiter plates (Dynex Technologies, Chantilly, Virginia, USA). Serial twofold dilutions of fusion protein $(50 \mu \mathrm{l})$, diluted in PBS- $0.5 \%$ nonfat dry milk, were reacted (2.5 hours, room temperature) with gentle rocking. After five washes with PBS, specific binding was detected with horseradish peroxidase-labeled anti-SA Ab (Zymed Laboratories Inc., South San Francisco, California, USA) $(50 \mu \mathrm{l}, 1: 3000$ dilution, 1 hour, room temperature). After three washes with PBS- $0.5 \%$ milk, the substrate ABTS $(50 \mu \mathrm{l})$ (Boehringer Mannheim Biochemicals Inc., Indianapolis, Indiana, USA) was added, and the OD was read at $405 \mathrm{~nm}$. The relative biotin-binding activity of SA-TNF- $\alpha$ fusion proteins was determined by comparison to a standard curve generated with wild-type SA (Zymed Laboratories Inc.) of known concentration.

Biotinylation of VLPs. BPV-1 L1 VLPs were generated from recombinant baculovirus-infected Sf9 cell cultures, as described previously (28). VLPs were incubated with NHS-LC-Biotin (Pierce Chemical Co.) at a $1: 2(\mathrm{wt} / \mathrm{wt}$ ) ratio (60 minutes, room temperature). To purify biotinylated VLPs, the mixture was layered onto a $24-54 \%$ linear sucrose gradient and centrifuged at $200,000 \mathrm{~g}$ for 3 hours in a SW- 40 rotor. Fractions were harvested from the bottom and analyzed by SDS-PAGE and Coomassie blue staining for the presence of L1. Positive fractions were pooled and then quantitated. The number of biotin molecules attached to each L1 molecule was determined using a spectrophotometric assay in which the displacement of 2-(4'-hydroxyazobenzene) benzoic acid (Pierce Chemical Co.) from avidin is measured upon the addition of biotinylated VLPs.

Conjugation of SA-TNF- $\alpha$ to particles. Biotinylated VLPs were incubated with recombinant SA or SA-TNF- $\alpha$ fusion protein at a 1:3 (wt/wt) ratio (1 hour, room temperature) with gentle rocking. Conjugated particles were separated from unconjugated SA using a MidJet cross-flow filtration system and MidGee cartridges with a 500-kDa pore size (A/G Technology Corp., Needham, Massachusetts, USA).

Inoculations. Antiserum was prepared by inoculating C57Bl $/ 6$ mice with $5 \mu \mathrm{g}$ wild-type BPV-1 L1 VLPs, $5 \mu \mathrm{g}$ VLPs conjugated to $15 \mu \mathrm{g}$ SA-TNF- $\alpha$ (conjugated VLPs were subsequently purified by cross-flow filtration), or $15 \mu \mathrm{g}$ unconjugated iminobiotin-purified SA-TNF- $\alpha$. Mice were inoculated intradermally three times at 
2-week intervals. In most cases, sera were collected at each injection and 2 weeks after the final boost. When CFA was used, antigen was diluted 1:1 in CFA for the initial injection and diluted 1:1 in incomplete Freund's adjuvant (IFA) for subsequent inoculations. Vaccinations were also performed with Titermax Gold (CytRx Corp., Atlanta, Georgia, USA) at $20 \%$ of the total volume, or CpG oligonucleotide 1826 (30 $\mu$ g per injection) (29). Animal care was in accordance with the NIH guidelines.

ELISA analysis of Ab induction. Mouse Ab's to recombinant biologically active TNF- $\alpha$ (PeproTech Inc., Rocky Hill, New Jersey, USA) or SA (Zymed Laboratories Inc.) were detected by ELISA using 200 ng of the target protein per well. Mouse serum was serially diluted in PBS$0.5 \%$ milk and applied to wells. Reactivity to these target proteins was determined using horseradish peroxidase-labeled goat anti-mouse IgG (or IgM) (Boehringer Mannheim Biochemicals Inc.) at a dilution of 1:2,000 as a secondary Ab. Upon development, ODs were read at $405 \mathrm{~nm}$ by a ThermoMax microplate reader. $\mathrm{OD}_{405}$ values that were greater than twice background (usually $>0.1$ ) were considered positive.

Measurement of $A b$ avidity. The avidity index values of serum Ab's were determined by measuring the resistance of Ab-target complexes to $8 \mathrm{M}$ urea by ELISA. After the serum incubation, triplicate wells were treated with either PBS or $8 \mathrm{M}$ urea for 5 minutes. Subsequently, the wells were washed with PBS, and the ELISA was performed as described above. The avidity index was calculated as the ratio of the mean OD value of urea-treated wells to PBS control wells multiplied by 100. Avidity index values greater than $50 \%$ are designated as high avidity, between $30 \%$ and $50 \%$ are designated as intermediate avidity, and less than $30 \%$ are designated as low avidity, as described (30).

Collagen-induced arthritis. Seven-week-old male DBA/1 mice (The Jackson Laboratories, Bar Harbor, Maine, USA) were immunized intramuscularly with VLPs $(5 \mu \mathrm{g})$ or VLPs $(5 \mu \mathrm{g})$ conjugated to SA-TNF- $\alpha(3-22)$
$(-7.5 \mu \mathrm{g})$ in the presence of Titermax Gold (20\% of total volume). Identical booster injections were given at days 14,28 , and, in some instances, day 42 . One and four weeks after the final boost, the mice were immunized intradermally at the base of the tail with $200 \mu \mathrm{g}$ type II collagen isolated from bovine hyaline cartilage (Chemicon International, Temecula, California, USA) emulsified 1:1 in CFA (for the initial injection) or in IFA (for the second injection). Mice were examined for onset of CIA by examining each limb and assigning a clinical score, as follows: 0 , normal; 1 , slight swelling; 2, pronounced swelling; and 3, joint rigidity. Mice were followed for at least 6 weeks after the second collagen injection, except for arthritic mice, which were followed for 3 weeks after disease onset, at which time they were sacrificed. Significance of differences was examined using Student $t$ test or $x^{2}$ test. A $P$ value of less than 0.05 was considered to be significant. Sagittal sections of the proximal interphalangeal joint of the middle digit were examined by histology and immunohistochemistry. For immunohistochemistry, fixed sections were incubated with an anti-TNF- $\alpha$ mAb (MP6-XT22; PharMingen, San Diego, California, USA) at a 1:50 dilution and then stained using standard methods.

\section{Results}

Generation of SA-TNF- $\alpha$ fusion proteins. We took advantage of the strong interaction between biotin and SA to develop a flexible system allowing the biochemical conjugation of self-peptides onto preassembled VLPs (Figure 1a). Mouse TNF- $\alpha$ peptides were fused to the carboxy-terminus of a truncated form of SA using a plasmid and an expression system developed by Sano and Cantor (27). This segment of SA, which spans aa 16-135 of the mature protein, is less prone to aggregation than is the full-length protein, yet retains the ability to form tetramers and bind biotin (26). We generated three bacterially expressed SA proteins fused to mouse TNF- $\alpha$ peptides representing, respectively, aa

\section{Table 1}

IgG Ab titers against TNF- $\alpha$ and SA in vaccinated $C 57 \mathrm{Bl} / 6$ mice

\begin{tabular}{|c|c|c|c|c|}
\hline \multirow[b]{2}{*}{ Preparation ${ }^{\mathrm{B}}$} & \multirow[b]{2}{*}{ Adjuvant } & \multicolumn{2}{|c|}{ GMT ELISA titer ${ }^{A}$} & \multirow{2}{*}{$\begin{array}{l}\text { Ratio of titers } \\
\text { SA to TNF-O }\end{array}$} \\
\hline & & TNF- $\alpha$ & SA & \\
\hline 1. VLP:SA-TNF- $\alpha(3-22)^{C}$ & CFA & $5 \times 10^{4}$ & $7 \times 10^{4}$ & 1.4 \\
\hline 2. VLP:SA-TNF- $\alpha(3-54)^{\mathrm{D}}$ & CFA & $1.5 \times 10^{4}$ & $1.4 \times 10^{4}$ & 0.9 \\
\hline 3. VLP:SA-TNF- $\alpha(29-47)^{\mathrm{E}}$ & CFA & $<10$ & $2 \times 10^{4}$ & NA \\
\hline 4. VLP:SA-TNF- $\alpha(3-22)^{\mathrm{D}}$ & None & $1 \times 10^{3}$ & $6 \times 10^{3}$ & 6 \\
\hline 5. VLP:SA-TNF- $\alpha(3-22)^{F}$ & Titermax & $3 \times 10^{3}$ & $1.5 \times 10^{4}$ & 5 \\
\hline 6. VLP:SA-TNF- $\alpha(3-22)^{F}$ & CpG & $1 \times 10^{3}$ & $1 \times 10^{4}$ & 10 \\
\hline 7. $V L P+r T N F-\alpha^{E}$ & CFA & $<10$ & $<10$ & NA \\
\hline 8. VLP + TNF- $\alpha(3-22)^{\mathrm{E}, \mathrm{G}}$ & CFA & $<10$ & $<10$ & NA \\
\hline 9. SA-TNF- $\alpha(3-22)^{\mathrm{E}}$ & None & $<10$ & 20 & NA \\
\hline 10. SA-TNF- $\alpha(3-22)^{C}$ & CFA & 60 & $8 \times 10^{3}$ & 133 \\
\hline 11. VLP:SAE & CFA & $<10$ & $2 \times 10^{5}$ & NA \\
\hline
\end{tabular}

${ }^{\mathrm{A}} \mathrm{GMT}$ were calculated by end-point dilution ELISA, using recombinant full-length protein as the target antigen. ${ }^{\mathrm{B}} \mathrm{A}$ colon indicates the preparation was conjugated to biotinylated VLPs, a plus sign indicates that the preparation was mixed with wild-type VLPs. ${ }^{\mathrm{C}} n=6$. ${ }^{\mathrm{D}} n=5$. ${ }^{\mathrm{E}} n=3$. ${ }^{\mathrm{F}} n=2$. ${ }^{\mathrm{G} T N F}-\alpha(3-22)$, a synthesized peptide representing aa 3-22 of the mature form of mouse TNF- $\alpha$. CpG, CpG oligonucleotides; rTNF- $\alpha$, recombinant TNF- $\alpha$. 
a

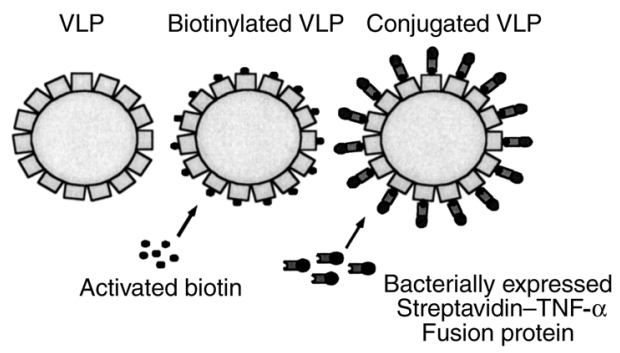

b

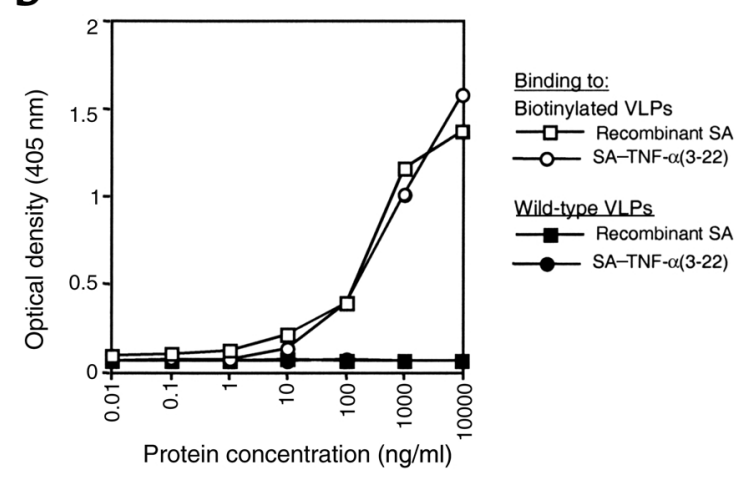

C

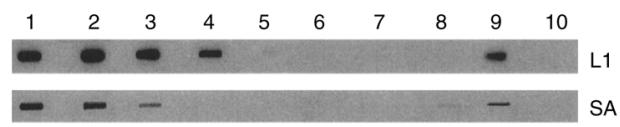

Figure 1

Binding of SA-TNF- $\alpha$ fusion protein to biotinylated VLPs. (a) Drawing summarizing conjugated vaccine construction. BPV L1 VLPs were coated with activated biotin and then purified on a linear sucrose gradient. Biotinylated particles were incubated with purified SA-TNF- $\alpha$ fusion protein to generate conjugated particles. (b) Binding of SA-TNF- $\alpha$ fusion protein to VLPs. Binding of recombinant SA or purified SA-TNF- $\alpha(3-22)$ to wild-type or biotinylated VLPs was measured by ELISA. (c) Cosedimentation of biotinylated VLPs with SA-TNF- $\alpha(3-22)$. Biotinylated VLPs were incubated with SA-TNF- $\alpha(3-22)$ and then separated on a $24-54 \%$ sucrose gradient. Gradient fractions were analyzed by Western blot analysis, using an anti-L1 mAb (mAB837) (top panel) or an anti-SA Ab (bottom panel). Fraction 1 represents the bottom and fraction 10 represents the top of the gradient.

$3-22,29-47$, and 3-54 of the mature protein. These mouse TNF- $\alpha$ peptides were chosen because they are homologous to regions of human TNF- $\alpha$ that were reported to interact with its receptor $(31,32)$.

The biotin-binding activity of the SA-TNF- $\alpha$ fusion proteins was assessed by ELISA using biotinylated VLPs as the target antigen. The TNF- $\alpha$ peptides had no effect on the ability of the fusion proteins to bind biotin (Figure $1 \mathrm{~b}$ and data not shown). The three fusion proteins bound biotinylated VLPs at a similar level as wild-type SA, and none bound to unbiotinylated VLPs. To determine the assembly state of the complexes, biotinylated VLPs were reacted with SA-TNF- $\alpha(3-22)$ and then subjected to analytical sucrose gradient centrifugation. Fractions were analyzed by slot-blot Western analysis using Ab's to BPV-1 L1 and SA. As shown in Figure 1c, SA-TNF- $\alpha(3-22)$ cosedimented with L1 in the higher molecular-weight fractions of the gradient, confirming that the fusion protein binds to biotinylated particles.

L1: SA-TNF- $\alpha$ stoichiometry in conjugated VLPs. To examine the location of the fusion protein on conjugated particles, we measured the ability of two well-characterized mAb's $(33,34)$ to bind conjugated particles by ELISA. Binding of an $\mathrm{Ab}$ that attaches to the sides of hexavalent capsomeres (mAb 5B6) was decreased by only twofold, relative to nonconjugated VLPs. However, conjugation led to a 10,000-fold decrease in the binding of $\mathrm{mAB} 9$, which binds to the tips of hexavalent and pentavalent capsomeres. These results suggest that the SA-fusion protein bound primarily to capsomere tips and that the exterior of the conjugated particles was well coated with fusion protein. Using a spectrophotometric assay to measure SA binding (35), we estimated that there are approximately $1.5 \mathrm{SA}$ tetramers bound per L1 molecule, which translates to approximately 540 tetramers per VLP (data not shown).

Humoral responses to conjugated VLPs. To examine the experimental induction of anti-TNF- $\alpha$ Ab's, groups of two to six C57Bl/ 6 mice were vaccinated with the SA-TNF- $\alpha$ fusion protein either alone or conjugated to biotinylated BPV-1 VLPs. Mice were vaccinated three times at 2 -week intervals. Mice that were immunized with conjugated particles received $5 \mu \mathrm{g}$ doses of VLPs that were bound with a saturating amount of SA-TNF- $\alpha$ fusion protein (representing approximately $7.5-10 \mu \mathrm{g}$ of protein). Mice injected with SA-TNF- $\alpha$ alone received doses of $15 \mu \mathrm{g}$ of the fusion protein. Control mice were inoculated with VLPs conjugated to recombinant SA or with unmodified VLPs mixed either with full-length recombinant mouse TNF- $\alpha$ or a chemically synthesized TNF- $\alpha$ peptide representing aa 3-22. Injections were performed both with and without selected adjuvants. Table 1 summarizes the anti-TNF- $\alpha$ and anti-SA geometric mean titers obtained with sera taken 2 weeks after the final boost as determined by IgG-specific ELISA using recombinant full-length TNF- $\alpha$ or SA, respectively, as the target antigen.

VLPs linked to SA-TNF- $\alpha(3-22)$ (Table 1 , line 1 ) or to SA-TNF- $\alpha(3-54)$ (line 2$)$ consistently induced hightiter anti-TNF- $\alpha$ Ab's. Conjugated SA-TNF- $\alpha(29-47)$ failed to generate anti-TNF- $\alpha$ Ab's (line 3 ), suggesting that the TNF- $\alpha$ domain of this polypeptide may be malfolded. Our analysis focused on particles conjugated to SA-TNF- $\alpha(3-22)$ because this preparation induced the highest autoantibody titers and it was easier to generate large quantities of this protein. VLPs conjugated to SA-TNF- $\alpha(3-22)$ generated hightiter IgG Ab responses even in the absence of exogenous adjuvant (Table 1, line 4; titers ranged from 640 to $10^{4}$ ), although CFA provided a 50 -fold boost to autoantibody levels (line 1 ; titers ranged from $10^{4}$ to $2 \times 10^{5}$ ). However, other adjuvants, including Titermax Gold (line 5) and CpG oligonucleotides (line 6), had little or no boosting effect on autoantibody titers.

In contrast to the strong anti-TNF- $\alpha$ response induced when the TNF- $\alpha$ peptide was conjugated to 


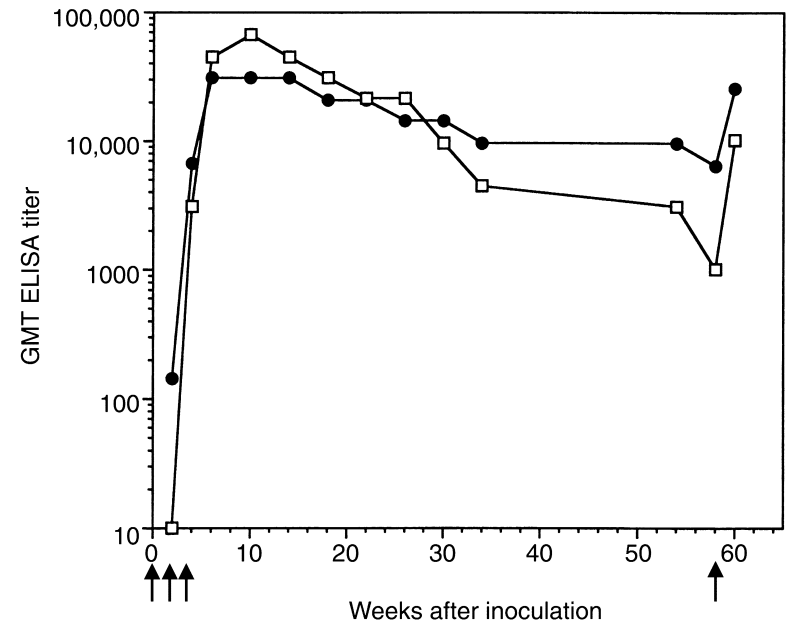

Figure 2

Serum IgG Ab titers in immunized C57Bl/6 mice. Anti-TNF- $\alpha$ (open squares) and anti-SA (filled circles) geometric mean titer (GMT) in sera from three mice immunized with VLPs conjugated to SA-TNF- $\alpha(3-22)$ and followed for over 1 year. Mice were immunized at weeks $0,2,4$, and 58 (indicated by arrows).

the VLPs, no IgG autoantibodies were detected when mice were inoculated with unfused TNF- $\alpha$ peptide or native TNF- $\alpha$ mixed with unlinked VLPs, even when the potent adjuvant CFA was used (Table 1, lines 7 and 8). Inoculation of SA-TNF- $\alpha(3-22)$ alone without adjuvant also failed to induce autoantibodies, and even SA-TNF- $\alpha(3-22)$ with CFA only induced low $\operatorname{IgG}$ autoantibody titers to TNF- $\alpha$ (lines 9 and 10 ; without CFA anti-TNF- $\alpha$ Abs were never detected, and with CFA titers they ranged from $<10$ to $10^{3}$ ). However, the immunizations that included linked foreign SA antigen induced high-titer IgG Abs to SA, which implies that the low or absent TNF- $\alpha$ Ab production seen with unconjugated SA-TNF- $\alpha(3-22)$ fusion protein was not the result of limiting Th activity. Therefore, when TNF- $\alpha$ was fused to a strong Th epitope(s), but not conjugated to the VLPs, the mouse immune system made a strong distinction between the foreign and self-polypeptides, with titers to SA that were more than 100-fold higher than those against TNF- $\alpha$. In contrast, conjugation to VLPs resulted in a 1,000-fold increase in autoantibody titers both with and without coadministration of adjuvant. This dramatic increase in autoantibody titer was accompanied by only modest changes in $\mathrm{SA} \mathrm{Ab}$ titers, resulting in similar $\mathrm{Ab}$ titers to TNF- $\alpha$ and SA (the ratio of SA to TNF- $\alpha$ titer ranged from 1.4 to 10 , depending on the adjuvant used). Taken together, the findings indicate that conjugation to VLPs was essential for high-titer IgG autoantibody induction and imply that when a selfantigen is displayed at high occupancy on a VLP surface, the humoral immune system loses its ability to distinguish between self and foreign.

To gain insight into the stage of the immune response that is modified by conjugation to VLPs, we measured anti-TNF- $\alpha$ IgM titers and compared them with the IgG titers. Table 2 compares the serum IgG data from 2 weeks after the third vaccination with IgM ELISA titers generated using sera taken 2 weeks after the initial vaccination. In contrast to IgG autoantibody responses, IgM titers to TNF- $\alpha$ were similar regardless of antigen preparation and the IgM titers to self (SA) and foreign (TNF- $\alpha$ ) epitopes were comparable for each formulation. Therefore, it does not appear that addition of linked VLPs initially increases the number of B cells that secrete Ig reacting with the self-peptide. Rather, VLP immunization may lead to amplification of the population of autoreactive mature B cells or may prevent these cells from being anergized.

High-titer autoantibodies persisted in immunized mice for months after vaccination. Three mice injected with VLPs conjugated to SA-TNF- $\alpha(3-22)$ were followed for over 1 year after immunization. Throughout this period of observation, none of the mice exhibited any obvious gross abnormalities. Ab titers against both SA and TNF- $\alpha$ slowly declined to levels that were approximately 5 -fold (SA) and 60-fold (TNF- $\alpha$ ) lower than peak titers (Figure 2). Thus, exposure to endogenous TNF- $\alpha$ does not appear to restimulate autoreactive B cells or to acutely anergize them. Autoantibody titers could be boosted by subsequent application of conjugated particles. At 58 weeks, the mice were given an additional injection of vaccine, and Ab titers versus SA and TNF- $\alpha$ increased fourfold and tenfold, respectively.

Protective effect of induced autoantibodies. To assess the protective potential of the induced TNF- $\alpha$ autoanti-

Table 2

Comparison of $\operatorname{lgM}$ and $\lg \mathrm{G}$ titers in vaccinated $\mathrm{C} 57 \mathrm{Bl} / 6$ mice

\begin{tabular}{|c|c|c|c|c|c|c|c|}
\hline \multirow[b]{2}{*}{ Preparation: } & \multirow[b]{2}{*}{ Adjuvant } & \multicolumn{3}{|c|}{ TNF- $\alpha$ ELISA GMTA } & \multicolumn{3}{|c|}{ SA ELISA GMT } \\
\hline & & $\underset{2 \text { weeks p.i. }}{\lg M}$ & $\begin{array}{l}\lg G \\
6 \text { weeks p.i. }\end{array}$ & $\begin{array}{c}\text { Ratio } \\
\text { IgG/IgM }\end{array}$ & $\underset{2 \text { weeks p.i. }}{\lg M}$ & $\begin{array}{c}\operatorname{lgG} \\
6 \text { weeks p.i. }\end{array}$ & $\begin{array}{c}\text { Ratio } \\
\text { IgG/IgM }\end{array}$ \\
\hline VLP:SA-TNF- $\alpha(3-22)$ & CFA & 320 & $5 \times 10^{4}$ & 150 & 200 & $7 \times 10^{4}$ & 350 \\
\hline VLP:SA-TNF- $\alpha(3-22)$ & None & 100 & $1 \times 10^{3}$ & 10 & 60 & $6 \times 10^{3}$ & 100 \\
\hline SA-TNF- $\alpha(3-22)$ & CFA & 90 & 60 & 0.6 & 30 & $8 \times 10^{3}$ & 267 \\
\hline VLP:SA & CFA & $<10$ & $<10$ & NA & 100 & $2 \times 10^{5}$ & 2500 \\
\hline
\end{tabular}

${ }^{A} G M T$ as described in Table 1. IgM titers were calculated by ELISA using sera isolated 2 weeks after the initial inoculation (p.i.). IgG titers were calculated using sera from the same mice, acquired 6 weeks after the initial inoculation ( 2 weeks after the third inoculation). 


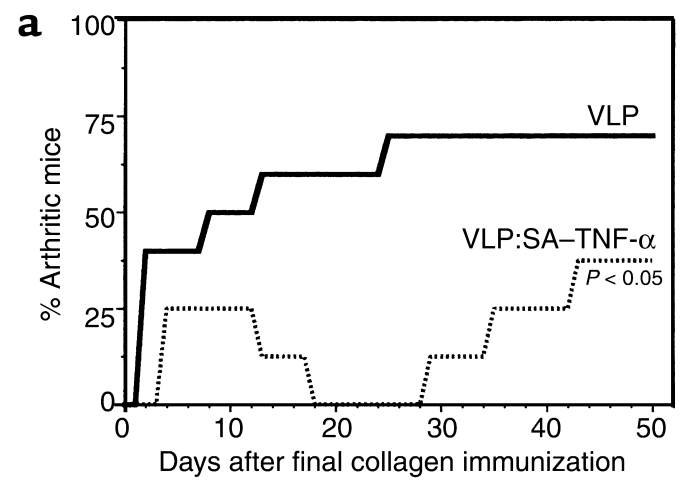

b

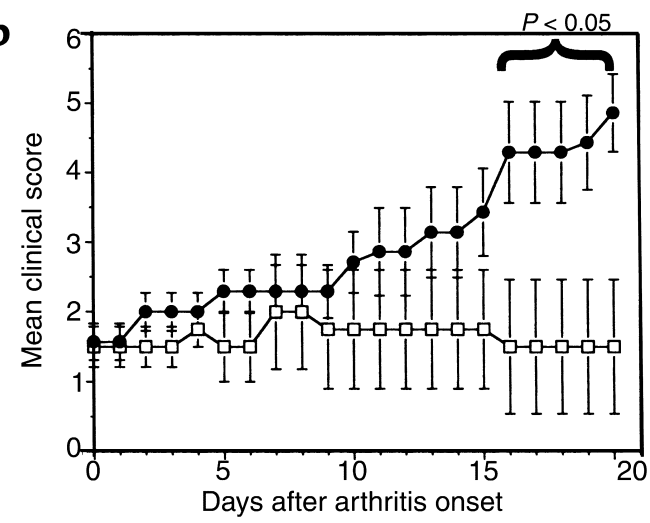

C
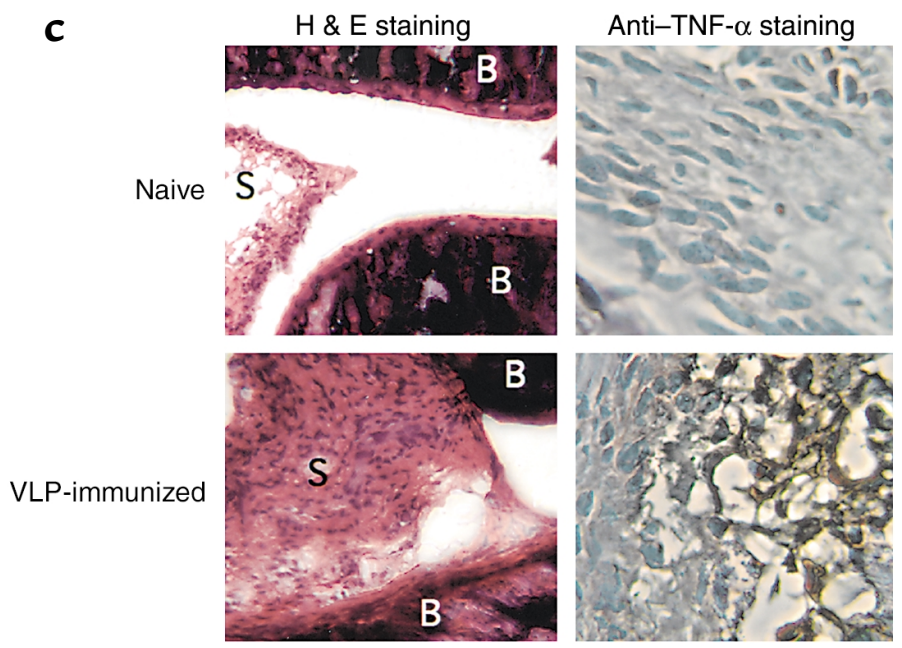

VLP:SA-TNF- $\alpha$
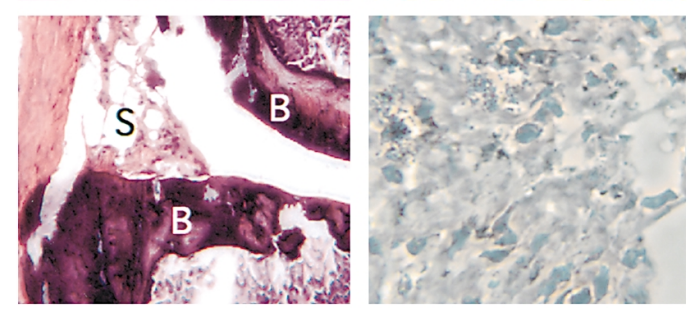

Figure 3

Immunization with conjugated VLPs ameliorates the symptoms of collagen-induced arthritis. DBA/1 mice were immunized four times with either VLPs conjugated to SA-TNF- $\alpha$ (3-22) or wild-type VLPs plus Titermax and then injected intradermally with bovine type II collagen plus Freund's adjuvant 1 and 3 weeks after the final vaccination. (a) Percentage of arthritic mice; groups immunized with wild-type VLPs (solid line) or VLPs conjugated to SA-TNF- $\alpha$ (3-22) (dashed line). (b) Clinical score: VLP-immunized mice (filled circles; VLP:SA-TNF- $\alpha(3-22)$-immunized mice (open squares). Clinical score data is for arthritic mice only. Error bars reflect SEM. (c) Representative joints taken from naive or collagen-injected mice immunized with either wild-type VLPs or VLPs conjugated to SA-TNF- $\alpha(3-22)$. Joints were taken 4 weeks after the second collagen injection. The first column of panels shows hematoxylin and eosin (H\&E) staining of synovial joints. $\times 10$. B, bone; $S$, synovial membrane. The second column of panels shows representative immunohistochemical analysis of TNF- $\alpha$ expression in each set of mice. $\times 40$. The percentage of TNF- $\alpha$-positive cells in joints from protected vaccinated mice was similar to that in naive mice.

bodies, we examined the effects of conjugated VLP vaccination on the development of type II collagen-induced arthritis (CIA) in mice. A total of $15 \mathrm{DBA} / 1$ mice were immunized with conjugated VLPs in two separate experiments. In the first experiment, seven mice were injected three times at 2-week intervals with $5 \mu \mathrm{g}$ of VLPs maximally conjugated to SA-TNF- $\alpha(3-22)$ in Titermax adjuvant. Five control mice were injected on the same schedule with the same dose of wild-type VLPs. At 1 week and 4 weeks after the final vaccination, mice were inoculated with $200 \mu \mathrm{g}$ bovine type II collagen. In mice vaccinated with VLP:SA-TNF- $\alpha$, the incidence of arthritis was apparently reduced to $71 \%$ (five of seven), compared with $100 \%$ in the control group (five of five). Protection was found to correlate with anti-TNF- $\alpha$ Ab titers (Table 3, experiment 1$)$. The three mice with the highest titers $\left(10^{4}\right)$ were either fully protected (two of three) or had a delayed onset of disease, whereas all four mice with low $\mathrm{Ab}$ titers $\left(10^{3}\right)$ rapidly developed disease at a rate that was not significantly different from control mice (mean, 15 days after collagen immunization).
These data suggested that protection might be improved by increasing TNF- $\alpha \mathrm{Ab}$ titers. To that end, a second experiment was initiated in which mice were given an additional boost of conjugated particles before collagen injection. Eight mice were immunized four times at 2-week intervals with VLPs conjugated to SA-TNF- $\alpha(3-22)$ in Titermax, along with ten VLP-injected control mice. This immunization schedule resulted in six of eight mice with anti-TNF- $\alpha$ titers of $3 \times 10^{3}$ or higher (Table 3). However, there was greater heterogeneity in the avidity of the Ab's than in experiment 1 . As above, type II bovine collagen was administered 1 and 4 weeks after the final vaccination. Vaccination resulted in a reduction of disease incidence and severity, relative to controls (Figure 3). In the vaccinated mice that did develop persistent arthritis, disease was delayed (Figure 3a). While none of the vaccinated mice developed the most severe disease, four of ten control mice displayed complete joint rigidity (clinical score, 3 ) in at least one limb. Five of the eight TNF- $\alpha$-vaccinated mice were healthy at the end of the study, although one of the healthy mice 
experienced transient swelling in one limb. In comparison, $70 \%$ (seven of ten) of the control mice developed arthritis $(P<0.05)$. Histological examination of joints from protected vaccinated mice confirmed the clinical data; these mice displayed a marked reduction in synovitis and joint erosion relative to control mice (Figure $3 c)$. Protection from arthritis induction was associated with both $\mathrm{Ab}$ titer and avidity (Table 3 ). All four mice with high avidity Ab's (i.e., those with an avidity index score $>50 \%$ ) remained healthy, whereas the mice with lower avidity Ab's (<50\%) developed transient disease or became arthritic. Correspondingly, vaccination with conjugated particles gave substantial reduction in the proportion of cells staining positive for TNF- $\alpha$ expression in the joints of protected mice (Figure $3 c$ ).

We also analyzed the results after combining the data from the two experiments. Within the vaccinated group, the geometric mean autoantibody titer in mice from both experiments that did not develop arthritis was 8,400 , and the mean avidity index was $62 \%$, compared with 1,300 and $44 \%$, respectively, in mice that developed arthritis. The conjugated particle vaccine reduced the incidence of arthritis from $80 \%$ in the control group (12 of 15$)$ to $53 \%$ in the vaccinated mice (8 of $15, P<0.05)$. However, substantially better protection was observed in mice with anti-TNF- $\alpha$ Ab titers over 3,000; only two of nine mice $(22 \%, P<0.0001)$ in this group developed disease, and both of the sick mice had avidity index values less than 50\%.

\section{Discussion}

Here we demonstrate that a TNF- $\alpha$ peptide when conjugated to a papillomavirus VLP can elicit high-titer autoantibody responses in mice that protect against disease in an experimental arthritis model that is partially TNF- $\alpha$ dependent. The results reported here represent a technical advance in the ease with which selfantigens can be incorporated into VLPs, extend this approach to an in vivo disease model, and provide some insight into the mechanism by which VLPs abrogate B cell tolerance to self-antigens.

Due to the difficulty in generating chimeric self-peptide L1 proteins that would still assemble into VLPs, we have developed a potentially more flexible method for displaying a self-peptide in a polymeric repetitive structure by linking a SA-self-peptide fusion protein to preassembled biotinylated VLPs. To illustrate, our attempts to develop L1-primate CCR5 chimeric VLPs were unsuccessful, despite the fact that the primate CCR5 aa sequence we used was very similar to the mouse CCR5 peptide that was successfully incorporated into chimeric particles. However, using the conjugated VLP approach, we were successful at generating CCR5-conjugated VLPs and inducing high-titer antiCCR5 autoantibodies in pig-tailed macaques (our unpublished observations). The potential flexibility of the conjugation technology is a critical aspect if this strategy is to be expanded to induce autoantibodies against additional target self-antigens.

The results of this study support the concept that experimental induction of autoantibodies is a quantitative phenomenon, in which antigen structure, $\mathrm{T}$ cell help, and adjuvant play key roles in the magnitude of this response. Our results are consistent with previous animal studies in which coadministration of CFA, a very potent $\mathrm{B}$ cell stimulator, promotes induction of autoan-

Table 3

Immune responses and disease in immunized DBA/1 CIA mice

\begin{tabular}{|c|c|c|c|}
\hline Animal number & Avidity index $(\%)^{\mathrm{A}}$ & TNF- $\alpha$ titer ${ }^{B}$ & Disease status $^{C}$ \\
\hline \multicolumn{4}{|l|}{ Experiment 1} \\
\hline 536 & 46 & $1 \times 10^{4}$ & Healthy \\
\hline 537 & 54 & $1 \times 10^{3}$ & Arthritic at day 8 \\
\hline 540 & 52 & $1 \times 10^{3}$ & Arthritic at day 14 \\
\hline 542 & 45 & $1 \times 10^{4}$ & Healthy \\
\hline 543 & 49 & $1 \times 10^{3}$ & Arthritic at day 8 \\
\hline 544 & 48 & $1 \times 10^{4}$ & Arthritic at day 35 \\
\hline 545 & 53 & $1 \times 10^{3}$ & Arthritic at day 10 \\
\hline \multicolumn{4}{|l|}{ Experiment 2} \\
\hline 881 & 73 & $1 \times 10^{4}$ & Healthy \\
\hline 882 & 61 & $1 \times 10^{4}$ & Healthy \\
\hline 883 & 25 & $1 \times 10^{2}$ & Arthritic at day 43 \\
\hline 885 & 44 & $1 \times 10^{3}$ & Transient disease days $4-7$, arthritic at day 3 \\
\hline 886 & 81 & $3 \times 10^{3}$ & Healthy \\
\hline 887 & 33 & $1 \times 10^{4}$ & Transient disease days $4-13$, then healthy \\
\hline 888 & 34 & $1 \times 10^{4}$ & Arthritic at day 29 \\
\hline 890 & 95 & $3 \times 10^{3}$ & Healthy \\
\hline
\end{tabular}

ADetermined by measuring the relative stability of the TNF- $\alpha$-Ab complex to an $8 \mathrm{M}$ urea wash in an ELISA. Values were calculated by using the equation (ureawashed wells/PBS-washed wells) $\times 100$, where values $x \geq 50 \%, 50 \%>x>30 \%$, and $x \leq 30 \%$ have been defined as high, intermediate, and low avidity, respectively. ${ }^{B}$ As described in Table 1. CMice were considered persistently arthritic if they had at least one arthritic limb (clinical score $\geq 1$ ) for at least 2 weeks. Two mice developed transient disease. One of these mice remained healthy, the other developed persistent arthritis 4 weeks after the transient disease resolved. 
tibodies to recombinant proteins containing foreign T-helper epitopes $(13,15,36,37)$. However, we found that presentation of a self-peptide in an ordered array that mimics the surface of a naked icosahedral virion could induce high-titer autoantibodies in the absence of conventional adjuvants. Furthermore, presentation to the immune system in this assembled state markedly diminished the distinction between self and foreign in a way that was not mimicked by CFA. While CFA acted synergistically with VLP conjugation for the induction of autoantibodies, conjugation to VLPs was the key factor in markedly enhancing Ab titers to self 1,000-fold relative to mice injected with self-antigen linked to foreign Th epitopes alone. Unfortunately, two other adjuvants that would likely be more suitable for use in humans, TiterMax Gold and CpG oligonucleotides, had little, if any, effect on boosting Ab titers to conjugated VLPs. A future challenge will be to identify better-tolerated adjuvants that are as effective as CFA at complementing VLPs for autoantibody production.

It is uncertain how display of self-epitopes on VLPs abrogates humoral immune distinction between self and foreign. It is well established that the ordered display of virion surface epitopes promotes $\mathrm{Ab}$ responses to these viral determinants, even in transgenic mice that constitutively express the virion protein (17). It has been proposed that repetitive determinants at a spacing of 5-10 nm, as found on papillomavirus VLPs, are unique to microbial antigens, and the mammalian immune system has evolved to respond strongly to this arrangement of epitopes (38-41). In contrast to IgG responses, $\operatorname{IgM}$ responses to self and foreign determinants were similar in our study, regardless of whether the fusion protein was linked to VLPs. This observation suggests that conjugation to VLPs results in a signal that promotes proliferation or survival of mature IgG-secreting $\mathrm{B}$ cells. One possibility is that this signal is transmitted through oligomerization of the specific $B$ cell receptors that recognize the self-peptide. Alternatively, this signal may be transmitted through a virion-specific receptor on the B cells or perhaps through a trans-acting signal triggered by VLP engagement of other immune regulatory cells. Dendritic cells are one possible mediator, as VLPs induce acute activation of bone marrow-derived dendritic cells (DCs) (42), and DCs play a role in B cell differentiation and isotype switching (43). It seems unlikely that enhancement of linked Th responses is the critical factor responsible for increased autoantibody response after conjugation to VLPs, since high-titer IgG responses to the SA portion of the fusion protein could be generated without linkage to the VLPs.

Vaccination with conjugated particles reduced the incidence and severity of disease in a mouse CIA model. The mechanism of protection is not clear, as the induced TNF- $\alpha$ autoantibodies were only weakly neutralizing in an in vitro assay (data not shown). However, our results in this disease model strongly suggest that Ab's provide the basis for protection, as efficacy of the vaccine was dependent on the titer and avidity of the autoantibodies produced. Furthermore, in vitro stimulation of spleen cells isolated from vaccinated mice with TNF- $\alpha$ peptide failed to induce IFN- $\gamma$ production (whereas stimulation of cells with VLPs or SA induced IFN- $\gamma$ ), suggesting that immunized mice do not mount appreciable $\mathrm{T}$ cell responses to the autoantigen (our unpublished observations). The level of protection reported here is comparable to that obtained in a previous study using an anti-TNF- $\alpha \mathrm{mAb}$ in the same experimental model, albeit in a therapeutic context (44). Inhibition of disease development in this model was also recently reported using a prophylactic vaccine consisting of a chimeric full-length TNF- $\alpha$ protein containing an inserted well-defined mouse Th epitope (13). However, many injections of relatively high doses of protein were required to produce a protective effect, and the use of CFA in that study would preclude direct translation of the vaccination protocol into human trials. In the current study, protection was induced using the less-inflammatory adjuvant Titermax, and similar levels of autoantibodies were elicited without adjuvant. Papillomavirus VLPs have proven to be highly immunogenic and well tolerated in clinical trials $(20,21)$. Taken together, our findings support the idea that autoantibody-inducing vaccines based on conjugated VLPs would be better tolerated and substantially more immunogenic than previously reported formulations.

The potential safety of long-term circulating self-reactive Abs must be carefully assessed in considering the potential therapeutic utility of autoantibody-inducing vaccines. In contrast to $\mathrm{mAb}$-based therapies, it would not be possible to rapidly decrease the serum Ab levels in response to adverse side effects. In our study we observed that TNF- $\alpha$ autoantibodies did slowly decline, suggesting that the endogenous TNF- $\alpha$ did not boost (or rapidly anergize) the autoreactive $B$ cells elicited by vaccination. These mice remained responsive; a booster vaccination over 1 year after the initial dose effectively elevated autoantibody titers. It is likely that the safety and therapeutic efficacy of this type of vaccine will vary greatly depending upon the disease and protein target, and these parameters will need to be investigated thoroughly in animal models for each potential target. With regard to TNF- $\alpha$, it is encouraging that no gross adverse effects were noted in the TNF- $\alpha$ autoantibody-producing mice that were followed for over 1 year. It is also noteworthy that TNF- $\alpha$ mAb therapy has proven sufficiently safe and effective clinically against rheumatoid arthritis and Crohn disease to warrant U.S. Food and Drug Administration approval. If safety issues can be adequately addressed, autoantibody-inducing vaccines may prove to be attractive alternatives to mAb's for these and other chronic diseases for which long-term therapy is likely to be required.

\section{Acknowledgments}

We thank P. Lenz, D. Pastrana, and S. Jay for assistance with mice, C. Cantor and T. Sano for supplying the SA expression vector, and A. Krieg for providing $\mathrm{CPG}$ oligonucleotides. 
1. Pegram, M., and Slamon, D. 2000. Biological rationale for HER2/neu (cerbB2) as a target for monoclonal antibody therapy. Semin. Oncol. 27:13-19.

2. Schenk, D., et al. 1999. Immunization with amyloid- $\beta$ attenuates Alzheimer-disease-like pathology in the PDAPP mouse. Nature. 400:173-177.

3. Chang, T.W. 2000. The pharmacological basis of anti-IgE therapy. Nat. Biotechnol. 18:157-162.

4. Papavasiliou, F., et al. 1997. V(D)J recombination in mature B cells: a mechanism for altering antibody responses. Science. 278:298-301.

5. Hertz, M., and Nemazee, D. 1998. Receptor editing and commitment in B lymphocytes. Curr. Opin. Immunol. 10:208-213.

6. Han, S., et al. 1997. V(D)J recombinase activity in a subset of germinal center B lymphocytes. Science. 278:301-305.

7. Albert, L.J., and Inman, R.D. 1999. Molecular mimicry and autoimmunity. N. Engl. J. Med. 341:2068-2074.

8. Youssef, S., et al. 2000. C-C chemokine-encoding DNA vaccines enhance breakdown of tolerance to their gene products and treat ongoing adjuvant arthritis. J. Clin. Invest. 106:361-371.

9. Wildbaum, G., Youssef, S., and Karin, N. 2000. A targeted DNA vaccine augments the natural immune response to self TNF- $\alpha$ and suppresses ongoing adjuvant arthritis. J. Immunol. 165:5860-5866.

10. Ragupathi, G., et al. 2000. Induction of antibodies against GD3 ganglioside in melanoma patients by vaccination with GD3-lactone-KLH conjugate plus immunological adjuvant QS-21. Int. J. Cancer. 85:659-666.

11. Steinhoff, U., Burkhart, C., Arnheiter, H., Hengartner, H., and Zinkernagel, R. 1994. Virus or a hapten-carrier complex can activate autoreactive B cells by providing linked T help. Eur. J. Immunol. 24:773-776.

12. Talwar, G.P., et al. 1994. A vaccine that prevents pregnancy in women. Proc. Natl. Acad. Sci. USA. 91:8532-8536.

13. Dalum, I., et al. 1999. Therapeutic antibodies elicited by immunization against TNF- $\alpha$. Nat. Biotechnol. 17:666-669.

14. Dalum, I., Jensen, M.R., Hindersson, P., Elsner, H.I., and Mouritsen, S. 1996. Breaking of B cell tolerance toward a highly conserved self protein. J. Immunol. 157:4796-4804.

15. Richard, M., Grencis, R.K., Humphreys, N.E., Renauld, J.C., and Van Snick, J. 2000. Anti-IL-9 vaccination prevents worm expulsion and blood eosinophilia in Trichuris muris-infected mice. Proc. Natl. Acad. Sci. USA 97:767-772.

16. Chackerian, B., Lowy, D.R., and Schiller, J.T. 1999. Induction of autoantibodies to mouse CCR5 with recombinant papillomavirus particles. Proc. Natl. Acad. Sci. USA. 96:2373-2378.

17. Bachmann, M.F., et al. 1993. The influence of antigen organization on B cell responsiveness. Science. 262:1448-1451.

18. Baker, T.S., et al. 1991. Structures of bovine and human papillomaviruses. Analysis by cryoelectron microscopy and three-dimensional image reconstruction. Biophys. J. 60:1445-1456.

19. Trus, B.L., et al. 1997. Novel structural features of Bovine Papillomavirus capsid revealed by a three dimensional reconstruction to $9 \AA ̊$ resolution. Nat. Struct. Biol. 4:413-420.

20. Harro, C.D., et al. 2001. Safety and immunogenicity trial in adult volunteers of a human papillomavirus $16 \mathrm{~L} 1$ virus-like particle vaccine. J. Natl. Cancer Inst. 93:284-292.

21. Zhang, L.F., et al. 2000. HPV6b virus like particles are potent immunogens without adjuvant in man. Vaccine. 18:1051-1058.

22. Klareskog, L., and McDevitt, H. 1999. Rheumatoid arthritis and its animal models: the role of TNF- $\alpha$ and the possible absence of specific immune reactions. Curr. Opin. Immunol. 11:657-662.

23. Eigler, A., Sinha, B., Hartmann, G., and Endres, S. 1997. Taming TNF: strategies to restrain this proinflammatory cytokine. Immunol. Today. 18:487-492.

24. Maini, R.N., and Taylor, P.C. 2000. Anti-cytokine therapy for rheumatoid arthritis. Annu. Rev. Med. 51:207-229.

25. Mijatovic, T., Kruys, V., Caput, D., Defrance, P., and Huez, G. 1997. Interleukin- 4 and -13 inhibit tumor necrosis factor- $\alpha$ mRNA translational activation in lipopolysaccharide-induced mouse macrophages. J. Biol. Chem. 272:14394-14398.

26. Sano, T., and Cantor, C.R. 1991. Expression vectors for streptavidin-containing chimeric proteins. Biochem. Biophys. Res. Commun. 176:571-577.

27. Sano, T., and Cantor, C.R. 1990. Expression of a cloned streptavidin gene in Escherichia coli. Proc. Natl. Acad. Sci. USA. 87:142-146.

28. Kirnbauer, R., Booy, F., Cheng, N., Lowy, D.R., and Schiller, J.T. 1992. Papillomavirus L1 major capsid protein self-assembles into virus-like particles that are highly immunogenic. Proc. Natl. Acad. Sci. USA. 89:12180-12184.

29. Chu, R.S., Targoni, O.S., Krieg, A.M., Lehmann, P.V., and Harding, C.V. 1997. CPG oligodeoxynucleotides act as adjuvants that switch on $\mathrm{T}$ helper 1 (Th1) immunity. J. Exp. Med. 186:1623-1631.

30. Hedman, K., and Seppala, I. 1988. Recent rubella virus infection indicated by a low avidity of specific IgG. J. Clin. Immunol. 8:214-221.

31. Eck, M.J., and Sprang, S.R. 1989. The structure of tumor necrosis factor$\alpha$ at 2.5 Å resolution. J. Biol. Chem. 264:17595-17604.

32. Bloom, J.W., Bettencourt, J.D., and Mitra, G. 1993. Epitope mapping and functional analysis of three murine IgG1 monoclonal antibodies to human tumor necrosis factor- $\alpha$. J. Immunol. 151:2707-2716.

33. Booy, F.P., Roden, R.B.S., Greenstone, H.L., Schiller, J.T., and Trus, B.L. 1998. Two antibodies that neutralize papillomaviruses by different mechanisms show distinct binding patterns at $13 \AA$ A resolution. J. Mol. Biol. 281:95-106.

34. Roden, R.B.S., et al. 1994. Neutralization of bovine papillomavirus by antibodies to L1 and L2 capsid proteins. J. Virol. 68:7570-7574.

35. Green, N.M. 1965. A spectrophotometric assay for avidin and biotin based on binding dyes by avidin. Biochem. J. 94:23c-24c.

36. Ciapponi, L., et al. 1997. Induction of interleukin-6 (IL-6) autoantibodies through vaccination with an engineered IL-6 receptor antagonist. Nat. Biotechnol. 15:997-1001.

37. Kim, S.K., Ragupathi, G., Cappello, S., Kagan, E., and Livingston, P.O. 2000. Effect of immunological adjuvant combinations on the antibody and T-cell response to vaccination with MUC1-KLH and GD3-KLH conjugates. Vaccine. 19:530-537.

38. Bachmann, M.F., and Zinkernagel, R.M. 1997. Neutralizing antiviral B cell responses. Annu. Rev. Immunol. 15:235-270.

39. Bachmann, M.F., et al. 1997. The role of antibody concentration and avidity in antiviral protection. Science. 276:2024-2027.

40. Bachmann, M.F., and Zinkernagel, R.M. 1996. The influence of virus structure on antibody responses and virus serotype formation. Immunol. Today. 17:553-558.

41. Fehr, T., et al. 1997. Role of repetitive antigen patterns for induction of antibodies against antibodies. J. Exp. Med. 185:1785-1792.

42. Lenz, P., et al. 2001. Papillomavirus-like particles induce acute activation of dendritic cells. J. Immunol. 166:5346-5355.

43. Wykes, M., Pombo, A., Jenkins, C., and MacPherson, G.G. 1998. Dendritic cells interact directly with naive B lymphocytes to transfer antigen and initiate class switching in a primary T-dependent response. J. Immunol. 161:1313-1319.

44. Williams, R.O., Feldmann, M., and Maini, R.N. 1992. Anti-tumor necrosis factor ameliorates joint disease in murine collagen-induced arthritis. Proc. Natl. Acad. Sci. USA. 89:9784-9788. 